\title{
ENGINEERING AND TECHNOLOGY CHALLENGES FOR ACTIVE DEBRIS REMOVAL
}

\author{
J.-C. Liou \\ NASA Orbital Debris Program Office \\ 2101 NASA Parkway, Houston, Texas 77058, USA
}

\begin{abstract}
After more than fifty years of space activities, the near-Earth environment is polluted with man-made orbital debris. The collision between Cosmos 2251 and the operational Iridium 33 in 2009 signaled a potential collision cascade effect, also known as the "Kessler Syndrome," in the environment. Various modeling studies have suggested that the commonlyadopted mitigation measures will not be sufficient to stabilize the future debris population. Active debris removal (ADR) must be considered to remediate the environment. This paper summarizes the key issues associated with debris removal and describes the technology and engineering challenges to move forward.
\end{abstract}

\section{INTRODUCTION}

Fifty-four years after the launch of Sputnik 1, satellites have become an integral part of human society. Unfortunately, the ongoing space activities have left behind an undesirable byproduct - orbital debris. This environment problem is threatening the current and future space activities. For example, the U.S. Department of Defense's Joint Space Operations Center (JSpOC) issues about 30 conjunction warnings for operational spacecraft $(\mathrm{S} / \mathrm{C})$ on a daily basis. Based on JSpOC's conjunction warnings, more than 100 collision avoidance maneuvers were executed by satellite operators in 2010 to reduce the impact risks of their satellites with other objects in the U.S. Space Surveillance Network (SSN) catalog. The International Space Station (ISS) conducted 13 debris avoidance maneuvers between 1999 and 2011. Of the four known accident collisions between objects in the SSN catalog, the last one, the collision between Cosmos 2251 and the operational Iridium 33 in 2009, was the most significant. It was the first ever accidental catastrophic destruction of an operational satellite by another satellite. It also signaled the potential collision cascade effect in the environment, commonly known as the "Kessler Syndrome," predicted by Kessler and Cour-Palais in 1978 [1].

This is an Open Access article distributed under the terms of the Creative Commons Attribution License 2.0, which permits unrestricted use, distribution, and reproduction in any medium, provided the original work is properly cited. 


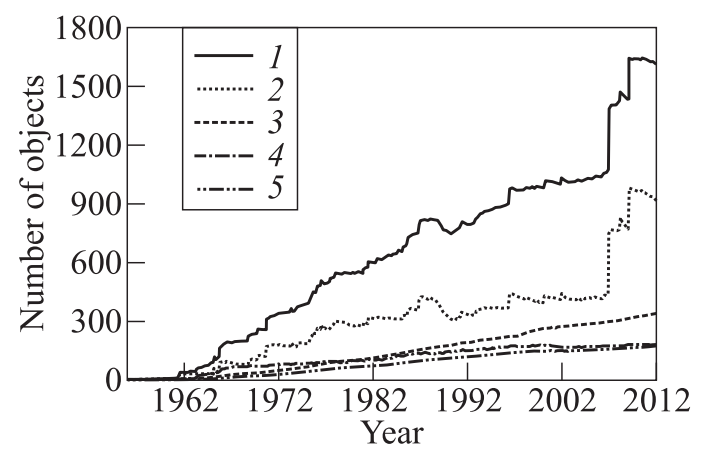

(a)

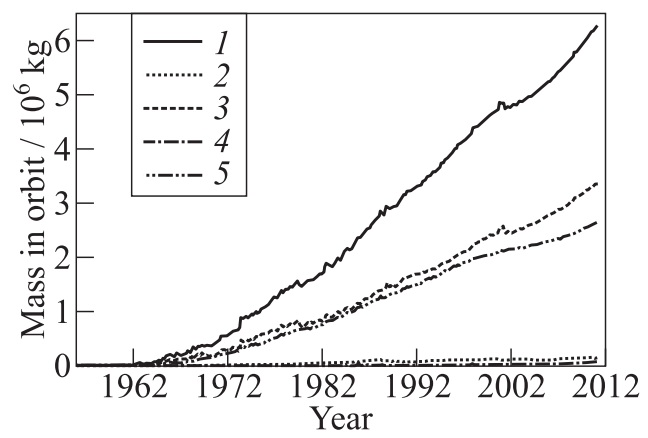

(b)

Figure 1 Increase of the historical SSN cataloged population through January 2012 (a) and historical mass increase of objects in Earth orbit (variations due to Shuttle missions are not included) $(b): 1-$ total objects; 2 - fragmentation debris; 3 $\mathrm{S} / \mathrm{C} ; 4-$ mission-related debris; and $5-\mathrm{R} / \mathrm{Bs}$

Figure $1 a$ shows the historical increase of objects in the SSN catalog. The majority of the cataloged objects are $10 \mathrm{~cm}$ and larger. The top curve is the total and the population breakdown is represented by the four curves below the total. As of January 2012, the SSN sensors were tracking more than 22,000 objects. However, approximately 6000 of them had yet to be fully processed and entered into the catalog. The catalogued population is heavily influenced by launches, new breakup events, and reentry of objects.

As shown in Fig. 1a, the total has been dominated by fragmentation debris throughout history. The two recent jumps correspond to the antisatellite (ASAT) test conducted by China in 2007 and the collision between Iridium 33 and Cosmos 2251 in 2009. Before the 2007 ASAT test, fragmentation debris were almost all explosion fragments. After these two major events, the ratio of collision fragments to explosion fragments was about one-to-one. It is expected 
that accidental collision fragments will further dominate the environment in the future.

The debris population does not stop at $10 \mathrm{~cm}$. Additional radar data indicate that at the $1 \mathrm{~cm}$ level the total is approximately 500,000. At the 1-millimeter level, the population is estimated to be on the order of hundreds of millions. Due to the high impact speed in space, even submillimeter debris pose certain safety concerns to satellite operators. The well-shielded U.S. modules of the ISS are protected against debris smaller than $1.4 \mathrm{~cm}$. For a typical operational S/C, however, a hypervelocity impact by debris $5 \mathrm{~mm}$ and larger is likely to lead to a mission-ending damage.

Figure $1 b$ depicts the historical increase of the on-orbit mass. Unlike the curves in Fig. 1a, which are heavily influenced by major breakups, the mass increase is relatively steady over time. The total mass is dominated by rocket

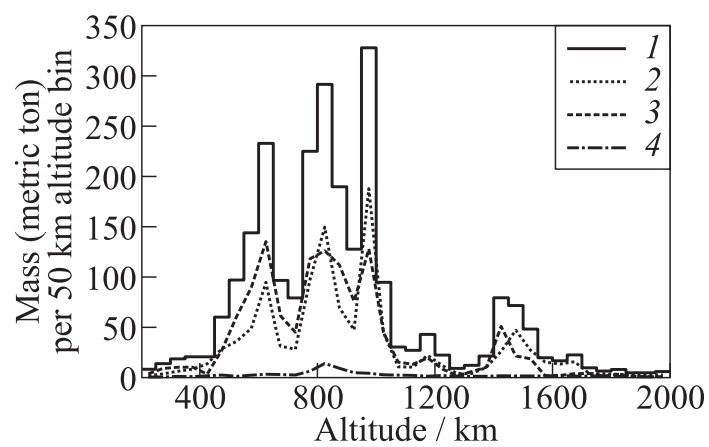

(a)

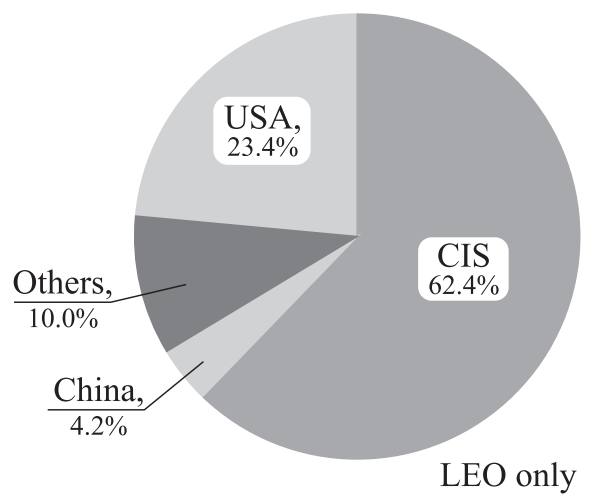

(b)

Figure 2 ( a) Mass distribution in LEO ( $1-$ all; $2-\mathrm{R} / \mathrm{Bs}$ ( $46 \%$ of all); $3-\mathrm{S} / \mathrm{C}$ (51\% of all); and 4 - others); and (b) sources of the LEO mass 
bodies $(\mathrm{R} / \mathrm{Bs})$ and $\mathrm{S} / \mathrm{C}$. They combine for approximately $96 \%$ of the mass in orbit. Although more than half of the cataloged objects are fragmentation debris, they only account for less than $3 \%$ of the total mass in space. From the trend of the total, there is no sign of slowing down. This is a major problem for the environment. As one continues to add more mass to the environment, it will only fuel the potential for the collision cascade effect.

The current total mass of materials orbiting the Earth is close to $6300 \mathrm{t}$, and $43 \%$ of it ( $2700 \mathrm{t}$ ) is in the low Earth orbit (LEO, the region below 2000-kilometer altitude). The distribution is not uniform (Fig. $2 a$ ). The three major peaks are located near 600, 800, and $1000 \mathrm{~km}$, respectively. Spacecraft and rocket bodies account for $97 \%$ of the mass in LEO. The 600 -kilometer peak is dominated by $\mathrm{S} / \mathrm{C}$ while the other two peaks are dominated by $\mathrm{R} / \mathrm{Bs}$. Source breakdown of the LEO mass is shown in Fig. $2 b$. Nearly $86 \%$ of the mass is in vehicles belonging to the Commonwealth of Independent States (CIS) and the Unites States.

The projected growth of the future debris population is shown in Fig. 3 . It is a summary of a study based on the LEGEND model developed by the NASA Orbital Debris Program Office. The study assumed nominal launches in the future, but no mitigation measures were implemented. In essence, the "no mitigation" assumption represents the worst-case scenario, but the results can be used to bound the future debris population projection. The environment is divided into three zones. The geosynchronous (GEO) region is within $200 \mathrm{~km}$ of the geosynchronous altitude. The region between LEO and GEO is defined as medium Earth orbit (MEO). Each projection curve is the average of 100

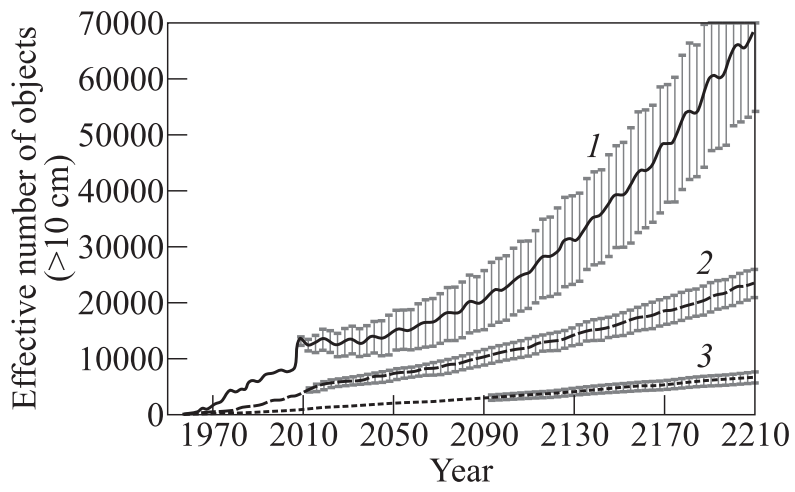

Figure 3 Projected growth of the $\geq 10$-centimeter populations in LEO, MEO, and GEO for the next 200 years. The simulations assumed nominal launches, but no mitigation measures were implemented in the future: 1 - LEO (200-2000-kilometer altitude); 2 - MEO (2000-35,586-kilometer altitude); and 3 - GEO (35,586-35,986-kilometer altitude) 
LEGEND Monte Carlo (MC) simulations. The 1- $\sigma$ standard deviation for each curve is also included in the figure.

The rapid increase of the debris population in LEO is a well-known trend [1]. It was the motivation for the development of the mitigation measures, such as passivation and the 25-year rule, by the international community in the last two decades. However, recent analyses have shown that the commonly-adopted mitigation measures will not be sufficient to stabilize the environment $[2,3]$. Therefore, to better limit the growth of the debris population in LEO additional measures, such as ADR, must be considered [4]. (The definition of ADR is to remove debris beyond the mitigation guidelines currently adopted by the international space community.)

The projected population growths in MEO and GEO over the next 200 years are not as severe as that in LEO. Even under the worst case scenario, nonmitigation assumption, the increase is very moderate. When postmission disposal (PMD) options, such as maneuvering satellites at the end of life to the graveyard orbit region, are implemented, the increase will be further reduced. Since there is no atmospheric drag to clean up the environment in MEO and GEO, the long-term build up of debris will continue. However, there is no urgent need to consider ADR in MEO and GEO in the near future.

\section{OPTIONS FOR ENVIRONMENT REMEDIATION}

There are different options for environment remediation in LEO. Several key questions must be addressed at the beginning to better focus the efforts. They include:

(1) Where is the most critical region for environment remediation?

(2) What are the short- and long-term mission objectives?

(3) What debris should be targeted first?

(4) What are the benefits to the environment?

(5) How to carry out the operations?

The answers to these questions will define the top-level requirements, drive the necessary technology development, and guide the implementation of the operations. In addition, nontechnical issues, such as policy, coordination, ownership, legal, and liability, at the national and international levels will also influence the direction of the planning and implementation.

Based on the projection curves shown in Fig. 3, it is obvious that any environment remediation efforts in the foreseeable future should focus on LEO. 


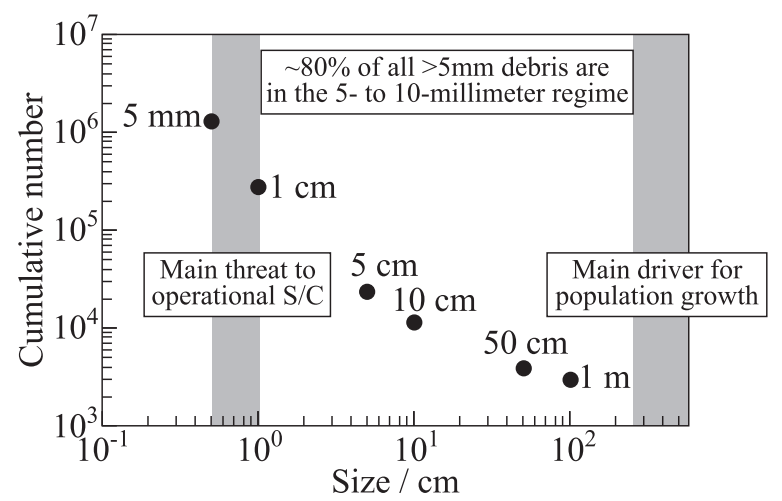

Figure 4 Cumulative size distribution of the LEO-crossing objects

Mission objectives will set the measures for success. Common mission objectives, such as maximizing the benefit-to-cost ratio and following practical mission constraints (in altitude, inclination, class, size, etc.) are always applicable to any ADR concepts. Specific mission objectives, on the other hand, are very diverse and will lead to very different forward paths. These objectives include, for example, controlling the LEO population growth (for small and large debris), limiting collision activities, mitigating short- or long-term risks (damage, not necessarily catastrophic destruction) for selected payloads, or mitigating risks for human space activities. Once a specific mission objective is selected, it needs to be further quantified (e.g., limiting the population growth or reducing mission-ending threat to a preset level) to better define the mission requirements.

What debris objects should be targeted first depends on the specific mission objective. A notional cumulative size distribution, plotted at the half-decade points, of the LEO-crossing objects is shown in Fig. 4. The population below $10 \mathrm{~cm}$ roughly follows a power-law size distribution - meaning there are far more smaller debris than larger ones. This means the main mission-ending threat for operational S/C in the environment comes from debris just above the threshold of the vehicle's impact protection shields. Since S/C all have different configurations and shielding designs, the "critical debris size" varies from S/C to S/C. For most operational $\mathrm{S} / \mathrm{C}$, any impact by debris larger than $5 \mathrm{~mm}$ is likely to cause mission-ending damage. The chances of similar damage diminish if the $\mathrm{S} / \mathrm{C}$ is impacted by smaller debris.

Based on the size distribution of Fig. 4, debris in the 5- to 10-millimeter regime represent about $80 \%$ of all objects larger than $5 \mathrm{~mm}$. Therefore, if the goal is to reduce the mission-ending threat for most operational $\mathrm{S} / \mathrm{C}$, then the remediation efforts should focus on $\mathrm{ADR}$ of the 5- to 10-millimeter debris. If 
the mission objective is to limit the growth of the future debris population or to reduce collision activities in the environment, then the remediation efforts should focus on the root cause of the problem - massive (one metric ton or more, at least several meters in dimension) $\mathrm{R} / \mathrm{Bs}$ and $\mathrm{S} / \mathrm{C}$ with high collision probabilities. Active removal of these objects from the environment is the most direct and effective long-term solution. The prevention of major debris-generating collisions involving massive intact objects may also be considered as a short-term option. However, an actionable collision prevention is predicated on several key factors, including accurate conjunction monitoring of all $\mathrm{R} / \mathrm{Bs}$ and retired $\mathrm{S} / \mathrm{C}$ in LEO, and since no mass is removed from the environment, this option is, at best, a temporary solution. The following sections will focus on the removal of small and massive/large debris and the associated challenges for the operations.

\subsection{Targeting the Main Threat for Operational Spacecraft}

The main challenges for removing 5- to 10-millimeter debris from LEO are related to the dynamic nature of the small debris and the huge number of their presence in the environment. The former is illustrated in Fig. 5, where a simulated evolution of the 5- to 10-millimeter Cosmos 2251 fragments between 2009 and 2019 is shown. The initial fragments were generated via the NASA Standard Breakup Model [5]. Individual fragments were then propagated forward in time, including Earth's $J_{2}, J_{3}, J_{4}$, solar-lunar gravitational perturbations, the solar radiation pressure, and the atmospheric drag. The National Oceanic and Atmospheric Administration (NOAA) solar flux F10.7 projection was combined with the Jacchia 1977 atmospheric model for the drag calculation [6]. Small debris tend to have high area-to-mass ratios (A/Ms). For those with perigees below about 1000-kilometer altitude, they are subjected to strong atmospheric drag perturbation. What the curves in Fig. 5 show is that, at any given altitude below $1000 \mathrm{~km}$, the 5- to 10-millimeter debris rapidly decay toward lower altitudes. At the same time, the region is rapidly replenished by debris spiraling down from higher altitudes. The environment is highly dynamic, and could have strong short-term (i. e., monthly to yearly) episodic variations.

The ISS example provided below outlines the technology challenges for removing small debris to significantly reduce the threat for a critical operational $\mathrm{S} / \mathrm{C}$. The ISS is constructed with the best micrometeoroid and orbital debris impact protection in history. The U.S. modules of the ISS are equipped with bumper shields that could withstand hypervelocity impacts by orbital debris $1.4 \mathrm{~cm}$ or smaller [7]. Since the ISS can conduct debris avoidance maneuvers against the catalogued objects per conjunction warnings provided by JSpOC, the main threat to the ISS comes from objects between 1.5 and $10 \mathrm{~cm}$. Cur- 


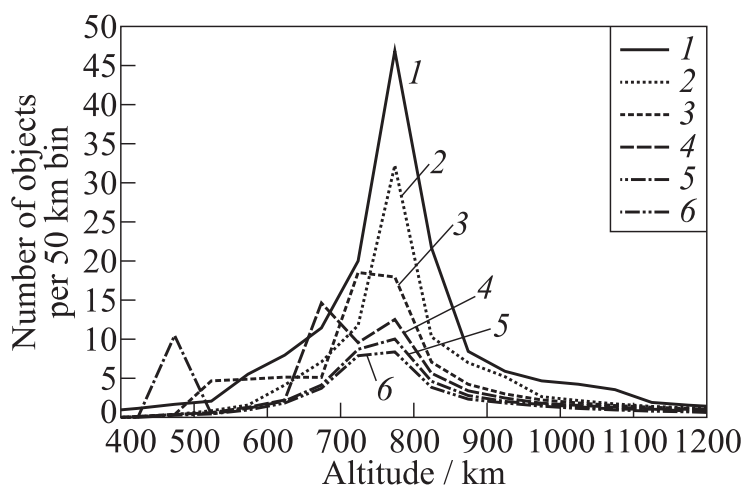

Figure 5 Altitude distributions of the simulated 5- to 10-millimeter Cosmos 2251 fragments between 2009 and 2019 (numbers in the parentheses indicate the total number of objects still in orbit): $1-2009(\sim 163,000) ; 2-2011(\sim 100,000) ; 3-2013$ $(\sim 87,000) ; 4-2015(\sim 67,000) ; 5-2017(\sim 59,000) ;$ and $6-2019(\sim 43,000)$

rently, the number of debris in this size range with orbits crossing the altitude of the ISS (330 to $360 \mathrm{~km}$ ) is approximately 1200 . Since these objects follow a power-law size distribution (see also Fig. 4), about 800 of them are between 1.5 and $3 \mathrm{~cm}$. If the ADR objective is to reduce the threat to the ISS by removing, for example, $50 \%$ of the debris in this size range, then trade studies must be conducted to investigate various options to meet the requirement.

Large-area debris collectors made of different materials and designed with different configurations have been proposed for small debris removal. The technology readiness levels (TRLs) for the commonly-mentioned capture mechanisms (e.g., using low density materials) are relatively mature. The key difficulty for the collector, however, is in the large area-time product that will be needed to remove any meaningful amount of small debris. Based on the estimated 1.5-to3 -centimeter debris flux at the ISS altitude, it will require a collector with an area-time product on the order of $1000 \mathrm{~km}^{2}$ year to remove 400 debris in this size range. For a one-year operation at the ISS altitude, the concept of a $1000 \mathrm{~km}^{2}$ cross-sectional area collector is simply not practical.

In addition, as illustrated in Fig. 5, the near ISS environment will continue to be replenished by debris decaying from higher altitudes. Any ADR operations of small debris, even if they are technically feasible and economically viable, will have to be carried out continuously for as long as the ISS remains active. Similarly, to remove any meaningful 5 - to 10 -millimeter debris at higher altitudes to better protect the majority of the operational S/C will be even more challenging because the demand for the area-time product will be significantly higher. 
A ground- or space-based laser system is another concept being proposed for the removal of millimeter-to-centimeter-sized debris. The unique technical challenges for this approach are the power required for the system, tracking capability for small debris, and the pointing accuracy of the laser system. Because of the concern for space weapons, this concept faces more nontechnical issues than others.

\subsection{Targeting the Root Cause of the Future Debris Population Growth}

The future debris population increase will be driven by fragments generated from accidental collisions involving large and massive $\mathrm{R} / \mathrm{Bs}$ or $\mathrm{S} / \mathrm{C}$ [8]. Therefore, the most effective way to limit the population growth is to remove the major "debris generators" from the environment. Major "debris generators" are those that have the highest collision probabilities with other objects in the environment, and when they do collide with other objects, they have the potential of generating the greatest amount of fragments. The latter is a function of mass. Hence, the mass, $M$, and the collision probability, $\mathrm{Pc}$, of each object can be used as a target selection criterion for the removal $[8,9]$. Numerical simulations have shown that, indeed, $[M \times \mathrm{Pc}]$ is an effective ADR selection criterion for environment remediation in $\operatorname{LEO}[4,8,10,11]$.

Figure 6 shows the effectiveness of debris population control of ADR based on the mass and collision probability criterion [12]. The historical environment

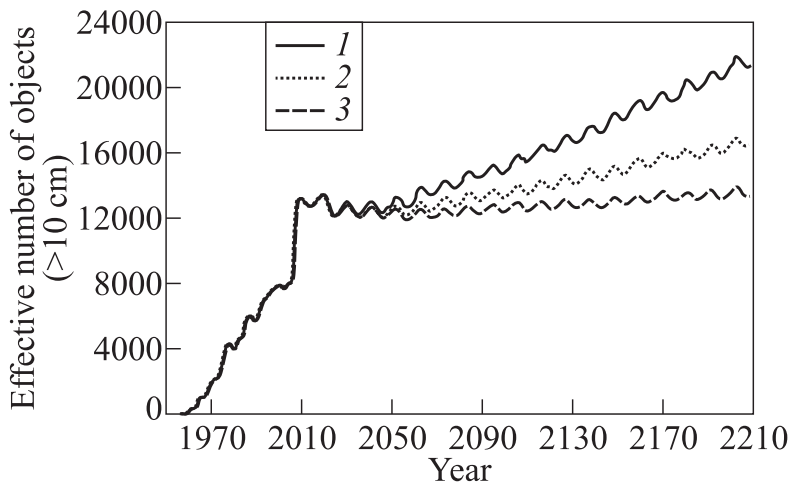

Figure 6 Projected future LEO populations (objects $\geq 10 \mathrm{~cm}$ ) based on three different scenarios. Each projection is the average of 100 LEGEND MC simulations: 1 regular launches + 90\% PMD; 2 - regular launches + 90\% PMD + ADR2020/02; 3 regular launches $+90 \%$ PMD + ADR2020/05 
includes fragments from the ASAT test and from the collision between Iridium 33 and Cosmos 2251. Each projection curve is the average of 100 LEGEND MC simulations. The scenario for the top curve assumes nominal launches and a $90 \%$ compliance of $\mathrm{PMD}$ for all R/Bs and $\mathrm{S} / \mathrm{C}$. The middle curve indicates that if an ADR of two objects per year is implemented after the year 2020 (denoted as ADR2020/02 in the figure), the population growth could be reduced approximately by half. If the ADR is increased to five objects per year (bottom curve), the population could be maintained at a level similar to the current environment. If a higher ADR rate is implemented, the future LEO environment can actually be better than what it is today.

The simulation results shown in Fig. 6 are intended to serve as a guide to illustrate the effectiveness of using $[M \times \mathrm{Pc}]$ as an ADR target selection criterion, to quantify how many objects need to be removed, and to show the benefits of ADR to the environment. The conclusion that "removing five objects per year can stabilize the LEO environment" is somewhat notional. A key assumption in the simulations is the nominal launches during the projection period. It is a common practice to repeat launches from the last 8 years for future environment simulations. If future launches are very different from the repeated cycle, including the launch frequency, mission orbits, and vehicle masses, then the required ADR rate will be somewhat different. Another assumption in the simulations is the immediate removal of objects from the environment. If that is not the case, then the required ADR rate will be higher. The 90 percent compliance assumption also has a direct impact to the projected population growth. The 90 percent compliance is significantly higher than the current reality. If the international space community cannot reach this level soon, future debris population will be worse than the top curve in Fig. 6, and it will certainly require a higher ADR effort to maintain the environment. Other factors, such as the long-term solar activity projection and practical operational constraints, will also affect the number of objects needing to be removed to stabilize the environment.

If the same $[M \times \mathrm{Pc}]$ criterion is applied to objects in the current LEO environment, potential targets for future ADR operations can be identified. Figure 7 shows their apogee/perigee altitudes and inclinations. It can be seen that many of the potential targets fall into several well-defined classes of vehicles between 600 and $1050 \mathrm{~km}$ altitudes, and in seven narrow inclination bands. These R/Bs and S/C have masses between 1 and 9 metric tons, and sizes up to more than 10 meters. The challenges for removing five such objects in a cost-effective manner on a yearly basis are monumental.

An end-to-end ADR operation includes many components - launch, ground support, propulsion, proximity operations, rendezvous, docking (capture or attachment), and finally, deorbit or graveyard maneuvers. From the cost perspective, multiple ADR systems per launch or secondary payload design are preferred. 


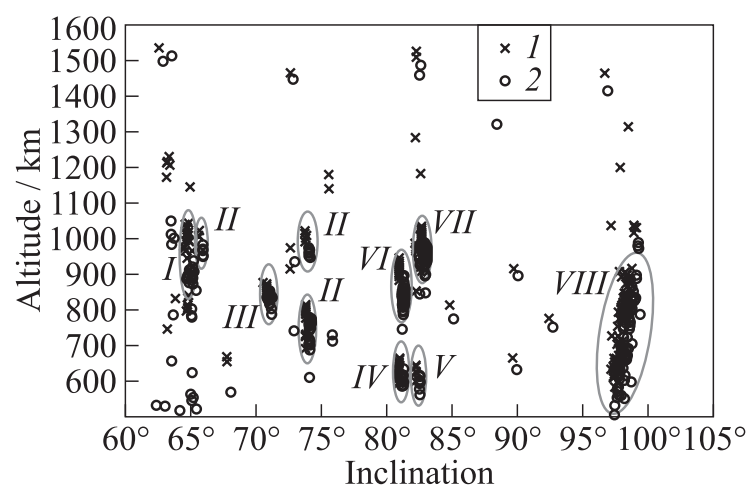

Figure 7 Altitude vs. inclination distribution of potential ADR targets: 1 - apogee; and 2 - perigee. Major classes of $\mathrm{R} / \mathrm{Bs}$ and $\mathrm{S} / \mathrm{C}$ are also labeled: $I$ - Cosmos $(1300 \mathrm{~kg}) ; I I-\mathrm{SL}-8 \mathrm{R} / \mathrm{B}(1400 \mathrm{~kg}) ; I I I-\mathrm{SL}-16 \mathrm{R} / \mathrm{B}(8900 \mathrm{~kg}), \operatorname{Cosmos}(3300 \mathrm{~kg}) ;$ $I V$ - Cosmos $(2500 \mathrm{~kg}) ; V-\operatorname{Cosmos}(2000 \mathrm{~kg}) ; V I-\mathrm{SL}-3 \mathrm{R} / \mathrm{B}(1440 \mathrm{~kg})$, Meteor $(2200-2800 \mathrm{~kg}) ; V I I$ - SL-8 R/B $(1400 \mathrm{~kg})$, Meteor $(2000 \mathrm{~kg})$; and VIII - various R/Bs and S/Cs (SL-16 R/B, Envisat, etc., 1000-8900 kg)

Options for propulsion, both for the ADR system vehicle and for the removal targets, are more diverse. The space tug concept based on chemical propellants is a mature technology, but the propellant mass and the operational cost may be too high for a routine operation. Electric propulsion can also be considered as an alternative. The concept of using electrodynamic tethers as a means for removing large orbital debris was first suggested in the 1990s [13]. Once the technology is mature and demonstrated, it could potentially provide a promising propellant-less option to deorbit ADR targets and to maneuver the ADR system from target to target. However, the collision risks of a long tether to other vehicles in the environment remain an open issue.

Attaching a drag enhancement device, such as an inflatable balloon or a thin-film sail, to a debris object is another potential low-cost option to deorbit massive ADR targets. The effectiveness of this concept is illustrated in Fig. 8. The orbital lifetime of an SL- 8 second stage, with a dry mass of $1400 \mathrm{~kg}$ and a 950-kilometer altitude, is more than 200 years. The addition of a lightweight and large-area device will increase the total $\mathrm{A} / \mathrm{M}$ of the system and cause it to decay more rapidly over time.

For example, a balloon with a diameter of about $30 \mathrm{~m}$ can deorbit the second stage in 25 years. If the size of the balloon is increased to $100 \mathrm{~m}$, then the orbital lifetime of the target can be reduced to just 2 years. The dimensions of the balloons, or equivalent thin-film sails, are not that unreasonable. However, as the system decays toward lower altitudes, its collision risks to other satellites in the environment will need to be evaluated as well. 


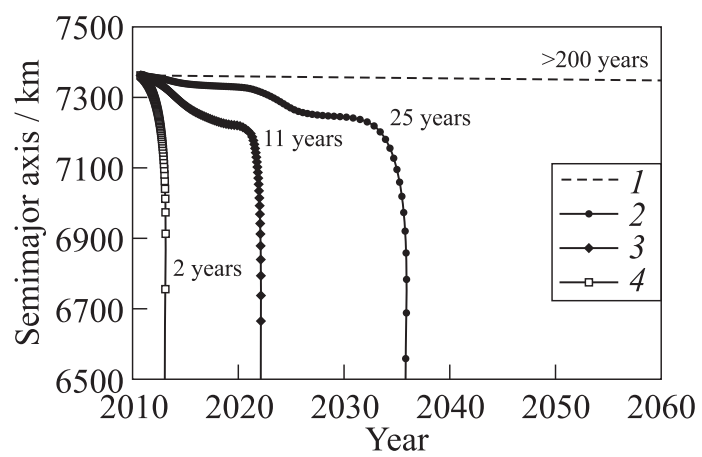

Figure 8 Effectiveness of deorbiting a typical 1400-kilogram SL-8 R/B from 950kilometer altitude with a drag enhancement device; a lightweight balloon (or an equivalent sail) with a diameter of $\sim 100 \mathrm{~m}$ can force the R/B to naturally decay in just 2 years: 1 -actual $\mathrm{A} / \mathrm{M}=0.015 \mathrm{~m}^{2} / \mathrm{kg} ; 2$ - enhanced $\mathrm{A} / \mathrm{M}=0.4 \mathrm{~m}^{2} / \mathrm{kg}, \sim 30$-meter balloon; 3 - enhanced $\mathrm{A} / \mathrm{M}=1.4 \mathrm{~m}^{2} / \mathrm{kg}, \sim 50$-meter balloon; and 4 - enhanced $\mathrm{A} / \mathrm{M}=5.5 \mathrm{~m}^{2} / \mathrm{kg}, \sim 100$-meter balloon

Proximity operations (including guidance, navigation, and control), rendezvous, and docking (capture or attachment) for ADR require new technologies as well because the targets are usually noncooperative and not designed for docking.

One potential major challenge is in the handling of the possible rapid spin/ tumble motion of the large and massive R/Bs and S/C. Some limited data seem to suggest that many of the potential ADR targets have tumble rates above $1 \mathrm{rpm}$. Ground-based radar or optical observations will be needed to survey the potential ADR targets to better characterize their tumble states and determine how these states might change over time. This is another area where new technologies may be needed for target stabilization if physical contact with the target is required during the removal operations.

Once a target is captured by the ADR system or is attached to a device, there are only two possible outcomes - a graveyard orbit or reentry. For some ADR concepts, it is possible to maneuver a high altitude (LEO) target to the graveyard orbit above 2000-kilometer altitude. This option is not a long-term solution. The cumulative debris mass eventually will create a new environment problem (via collisions) in the graveyard orbit and affect other operational regions. The best end result for an ADR operation is to bring the target down. However, the reentry risks of massive $\mathrm{R} / \mathrm{Bs}$ or $\mathrm{S} / \mathrm{C}$ must be assessed. If it is necessary to include a controlled reentry at the end, it may severely limit the ADR operational options and will significantly increase the overall cost. 


\section{CONCLUDING REMARKS}

The orbital debris problem is facing a critical point. The commonly-adopted mitigation measures will not be sufficient to fully control the debris population growth in LEO. As the international community gradually reaches a consensus on the need for ADR, the focus will shift from environment modeling to completely different challenges - technology development, systems engineering, and operations. As the community takes on these new challenges, a long-term strategic plan must be established first. Mission objectives must be clearly defined to develop a feasible forward path. If the goal is to remediate the environment, then four critical "Cs" will be needed at the international level. The first "C" stands for the consensus on ADR. The second "C" is for cooperation - the removal target may belong to a different country. The third " $\mathrm{C}$ " is for collaboration - it is highly unlikely that any single organization or country can accomplish the goal by itself. The last "C" stands for contributions — costsharing will be the key for using ADR to preserve the environment for future generations.

\section{REFERENCES}

1. Kessler, D., and B. Cour-Palais. 1978. Collision frequency of artificial satellites: The creation of a debris belt. JGR 83:2637-46.

2. Liou, J.-C., and N. Johnson. 2006. Risks in space from orbiting debris. Science 311:340-41.

3. Liou, J.-C., and N. Johnson. 2008. Instability of the present LEO satellite populations. Adv. Space Res. 41:1046-53.

4. Liou, J.-C., N. Johnson, and N. Hill. 2010. Controlling the growth of future LEO debris populations with active debris removal. Acta Astronautica 66:648-53.

5. Johnson, N., P. Krisko, J.-C. Liou, and P. Anz-Meador. 2001. NASA's new breakup model of EVOLVE 4.0. Adv. Space Res. 28:1377-84.

6. Jacchia, L. G. 1977. Thermospheric temperature, density, and composition: New model. Smithsonian Special Report SAO 375.

7. Hyde, J., H. Evans, K. Hoffman, E. Christiansen, D. Lear, and T. Prior. 2010. International Space Station micrometeoroid and orbital debris integrated threat assessment 12. NASA JSC-65837 Rev. A.

8. Liou, J.-C., and N. Johnson. 2007. A sensitivity study of the effectiveness of active debris removal in LEO. IAC-07-A6.3.05.

9. Liou, J.-C. 2006. Collision activities in the future orbital debris environment. Adv. Space Res. 38:2102-06. 
10. Liou, J.-C., and N. Johnson. 2009. A sensitivity study of the effectiveness of active debris removal in LEO. Acta Astronautica 64:236-43.

11. Lewis, H., G. Swinerd, R. Newland, and A. Saunders. 2009. Active removal study for on-orbit debris using DAMAGE. 5th European Conference on Space Debris Proceedings. ESA SP-672.

12. Liou, J.-C. 2011. An active debris removal parametric study for LEO environment remediation. Adv. Space Res. 47:1865-76.

13. Portree, D., and J. Loftus. 1999. Orbital debris: A chronology. NASA-TP-1999208856. 